\title{
Exploiting Symmetry in Channel Shortening Equalizers
}

\author{
Richard K. Martin, C. Richard Johnson, Jr., Ming Ding, and Brian L. Evans \\ Richard K. Martin and C. Richard Johnson, Jr. \\ Cornell University \\ School of Electrical \& Computer Engineering \\ Ithaca, NY 14853, USA \\ \{frodo, johnson\}@ece.cornell.edu \\ Ming Ding and Brian L. Evans \\ The University of Texas at Austin \\ Dept. of Electrical \& Computer Engineering \\ Austin, TX 78712-1084, USA \\ $\{$ ming, bevans\}eece.utexas.edu
}

Copyright 2002 IEEE. Published in the 2003 International Conference on Acoustics, Speech, and Signal Processing (ICASSP 2003), scheduled for April 6-10, 2003 in Hong Kong SAR, China. Personal use of this material is permitted. However, permission to reprint/republish this material for advertising or promotional purposes or for creating new collective works for resale or redistribution to servers or lists, or to reuse any copyrighted component of this work in other works, must be obtained from the IEEE. Contact:

Manager, Copyrights and Permissions

IEEE Service Center

445 Hoes Lane

P.O. Box 1331

Piscataway, NJ 08855-1331, USA.

Telephone: + Intl. 908-562-3966. 


\section{EXPLOITING SYMMETRY IN CHANNEL SHORTENING EQUALIZERS}

\author{
R. K. Martin and C. R. Johnson, Jr.* \\ Cornell University \\ School of Electrical and Computer Engineering \\ Ithaca, NY 14853, USA \\ \{frodo, johnson\}eece.cornell.edu
}

\author{
M. Ding and B. L. Evans ${ }^{\dagger}$ \\ The University of Texas at Austin \\ Dept. of Electrical and Computer Engineering \\ Austin, TX 78712-1084, USA \\ \{ming, bevans\}@ece. utexas.edu
}

\begin{abstract}
Time-domain equalization is crucial in reducing inter-carrier and inter-symbol interference in multicarrier systems. A channel shortening time-domain equalizer (TEQ), which is a finite impulse response (FIR) filter, placed in cascade with the channel produces an effective impulse response that is shorter than the channel impulse response. We show that finite length minimum mean squared error (MMSE) and maximum shortening SNR (MSSNR) TEQs are approximately symmetric, and infinite length MSSNR TEQs with a unit norm TEQ (UNT) constraint are exactly symmetric. A symmetric TEQ halves FIR implementation complexity, enables the frequency-domain equalizer and TEQ to be trained in parallel, and exhibits only a small loss in bit rate over nonsymmetric TEQs. In addition, a symmetric MSSNR-UNT TEQ reduces training computational complexity by a factor of 4 and doubles the length of the TEQ that can be designed.
\end{abstract}

\section{INTRODUCTION}

Multicarrier modulation (MCM) techniques such as orthogonal frequency division multiplexing (OFDM) and discrete multi-tone (DMT) have been receiving increasing attention in the literature recently, and they have been deployed in numerous industry standards. Applications include the wireless LAN standards IEEE 802.11a and HIPERLAN2; Digital Audio Broadcast (DAB) and Digital Video Broadcast (DVB) in Europe; and asymmetric and very-high-speed digital subscriber loops (ADSL, VDSL). MCM is attractive due to the ease with which it can combat channel dispersion, provided that the channel delay spread is not greater than the length of the cyclic prefix (CP). The cyclic prefix is a copy of the last $\nu$ samples of each symbol which is prepended to the start of each symbol in order to make the convolution of the data and channel appear periodic. However, if the CP

\footnotetext{
* This work was supported in part by NxtWave Communications (now ATI), Langhorne, PA.

${ }^{\dagger}$ This work was supported in part by The State of Texas Advanced Technology Program under project 003658-0614-2001.
}

is not long enough, the orthogonality of the sub-carriers is lost and this causes both inter-carrier interference (ICI) and inter-symbol interference (ISI).

A well-known technique to combat the ICI/ISI caused by an inadequate $\mathrm{CP}$ length is the use of a time-domain equalizer (TEQ). The TEQ is a finite impulse response filter that shortens the channel so that the delay spread of the combined channel-equalizer impulse response is not longer than the CP length. The TEQ design problem has been extensively studied in the literature [1] - [10]. This paper analyzes the MMSE [1] and MSSNR [4] TEQ design methods.

\section{SYSTEM MODEL AND NOTATION}

The multicarrier system model is shown in Fig. 1. Each block of bits is divided into $\frac{N}{2}$ bins, and each bin is viewed as a QAM signal that will be modulated by a different carrier. An efficient means of implementing the multicarrier modulation in discrete time is to use an inverse fast Fourier transform (IFFT). The IFFT converts each bin (which acts as one of the frequency components) into a time-domain signal. After transmission, the receiver can use an FFT to recover the data within a bit error rate tolerance, provided that equalization has been performed properly.

In order for the subcarriers to be independent, the convolution of the signal and the channel must be a circular convolution. It is actually a linear convolution, so it is made to appear circular by adding a cyclic prefix to the start of each data block. The cyclic prefix is obtained by prepending the last $\nu$ samples of each block to the beginning of the block. If the $\mathrm{CP}$ is at least as long as the channel, then the output of each subchannel is equal to the input times a complex scalar. The signals can then be equalized by a bank of complex gains, referred to as a frequency-domain equalizer (FEQ). If the channel is longer than $\nu+1$, a TEQ is needed to shorten the channel. We use the notation $\mathbf{h}, \mathbf{w}$, and $\mathbf{c}=\mathbf{h} \star \mathbf{w}$ to denote the channel, TEQ, and effective channel impulse responses, respectively; and $L_{w}$ denotes the TEQ length. 


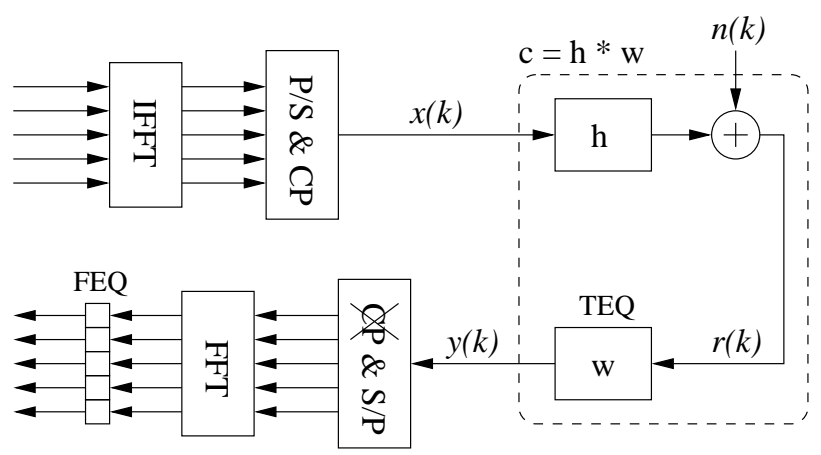

Fig. 1. System model. (I)FFT: (inverse) fast Fourier transform, P/S: parallel to serial, S/P: serial to parallel, CP: add cyclic prefix, $\mathrm{xCP}$ : remove cyclic prefix.

\section{THE TEQ IMPULSE RESPONSE}

This section shows that the MSSNR and MMSE designs often lead to TEQs with highly symmetric impulse responses. Section 3.1 reviews MSSNR and MMSE design methods. Section 3.2 shows why symmetry occurs in TEQ impulse responses, and Section 3.3 analyzes infinite-length MSSNR and MMSE TEQ designs.

\subsection{The MSSNR and MMSE solutions}

Consider the maximum shortening SNR (MSSNR) TEQ design [4]. This technique attempts to maximize the ratio of the energy in a window of the effective channel over the energy in the remainder of the effective channel. Define $\mathbf{H}_{\text {win }}$ and $\mathbf{H}_{\text {wall }}$ as in [4], so that $\mathbf{c}_{\text {win }}=\mathbf{H}_{\text {win }} \mathbf{w}$ yields a length $\nu+1$ window of the effective channel, and $\mathbf{c}_{\text {wall }}=\mathbf{H}_{\text {wall }} \mathbf{w}$ yields the remainder of the effective channel. The MSSNR design problem can be stated as [4], [11]

$$
\max _{\mathbf{w}}\left(\mathbf{w}^{T} \mathbf{B w}\right) \text { subject to } \mathbf{w}^{T} \mathbf{A} \mathbf{w}=1,
$$

where $\mathbf{A}$ and $\mathbf{B}$ are real, symmetric $L_{w} \times L_{w}$ matrices,

$$
\mathbf{A}=\mathbf{H}_{\text {wall }}^{T} \mathbf{H}_{\text {wall }}, \quad \mathbf{B}=\mathbf{H}_{\text {win }}^{T} \mathbf{H}_{\text {win }} .
$$

Solving (1) leads to a TEQ that satisfies the generalized eigenvector problem,

$$
\mathbf{B w}=\lambda \mathbf{A w} .
$$

The solution for $\mathbf{w}$ will be the generalized eigenvector corresponding to the largest generalized eigenvalue $\lambda$.

Iterative and adaptive implementations of the MSSNR approach have also been proposed. In [5], an iterative algorithm was proposed which performs a gradient descent of $\left\|\mathbf{c}_{\text {wall }}\right\|^{2}$. Although it is not mentioned in [5], this algorithm needs a constraint to prevent the trivial solution $\mathbf{w}=\mathbf{0}$. A natural constraint is to maintain $\|\mathbf{w}\|=1$ by renormalizing $\mathbf{w}$ after each iteration. A blind, adaptive algorithm was proposed in [9], which is a stochastic gradient descent on $\left\|\mathbf{c}_{\text {wall }}\right\|^{2}$, although it leads to a window size of $\nu$ instead of $\nu+1$. For these two algorithms, the solution must satisfy

$$
\min _{\mathbf{w}}\left(\mathbf{w}^{T} \mathbf{A w}\right) \text { subject to } \mathbf{w}^{T} \mathbf{w}=1 .
$$

This leads to a TEQ that must satisfy a traditional eigenvector problem,

$$
\mathbf{A} \mathbf{w}=\lambda \mathbf{w}
$$

The solution is the eigenvector corresponding to the smallest eigenvalue. We will refer to the solution for $\mathbf{w}$ in (3) as the MSSNR solution, and the solution of (5) as the MSSNR Unit Norm TEQ (MSSNR-UNT) solution.

When the input signal is white and there is no noise, the MMSE design produces the same TEQ as the MSSNR design [12]. This can be extended to the noisy case to show that the TEQ for the MMSE design must satisfy [13]

$$
\mathbf{B w}=\lambda\left(\mathbf{A}+\mathbf{R}_{n}\right) \mathbf{w},
$$

where $\lambda$ is the largest generalized eigenvalue of the matrix pair $\left(\mathbf{B},\left(\mathbf{A}+\mathbf{R}_{n}\right)\right)$. This allows for a unified treatment of the MSSNR and MMSE TEQ designs.

\subsection{Symmetry in eigenvectors}

Let $\mathbf{J}$ be the square matrix with ones on the cross diagonal, and zeros elsewhere. Symmetric centrosymmetric $N \times N$ matrices are defined as matrices in the set

$$
V_{N}=\left\{\mathbf{C}: \mathbf{C}^{T}=\mathbf{C}, \quad \mathbf{J C J}=\mathbf{C}\right\} .
$$

Symmetric centrosymmetric matrices of size $N \times N$ have exactly $\lceil N / 2\rceil$ symmetric eigenvectors and $\lfloor N / 2\rfloor$ skewsymmetric eigenvectors [14]. This result can be extended to the generalized eigenvector case.

Theorem 3.1 If $\mathbf{A}, \mathbf{B} \in V_{L_{w}}$ (so they are symmetric centrosymmetric) and $\mathbf{A}$ is invertible, then the eigenvectors of $\left(\mathbf{A}^{-1} \mathbf{B}\right)$ can always be chosen to be symmetric or skewsymmetric. Furthermore, if the eigenvalues of $\left(\mathbf{A}^{-1} \mathbf{B}\right)$ are distinct, then all of the eigenvectors will all be symmetric or skew-symmetric.

Proof: Since $\left(\mathbf{A}^{-1} \mathbf{B}\right)$ is centrosymmetric, $\mathbf{J}\left(\mathbf{A}^{-1} \mathbf{B}\right) \mathbf{J}=$ $\mathbf{A}^{-1} \mathbf{B}$. Thus, if $\mathbf{w}$ is an eigenvector of $\mathbf{A}^{-1} \mathbf{B}$, it satisfies

$$
\begin{aligned}
\left(\mathbf{J A}^{-1} \mathbf{B J}\right) \mathbf{w} & =\lambda \mathbf{w}, \\
\mathbf{A}^{-1} \mathbf{B}(\mathbf{J w}) & =\lambda(\mathbf{J w}), \\
\mathbf{A}^{-1} \mathbf{B}(-\mathbf{J} \mathbf{w}) & =\lambda(-\mathbf{J w})
\end{aligned}
$$

where we have made use of $\mathbf{J J}=\mathbf{I}$. Thus, if $\mathbf{w}$ is an eigenvector of $\left(\mathbf{A}^{-1} \mathbf{B}\right)$ with eigenvalue $\lambda$, then $\mathbf{J w}$ and $-\mathbf{J} \mathbf{w}$ 


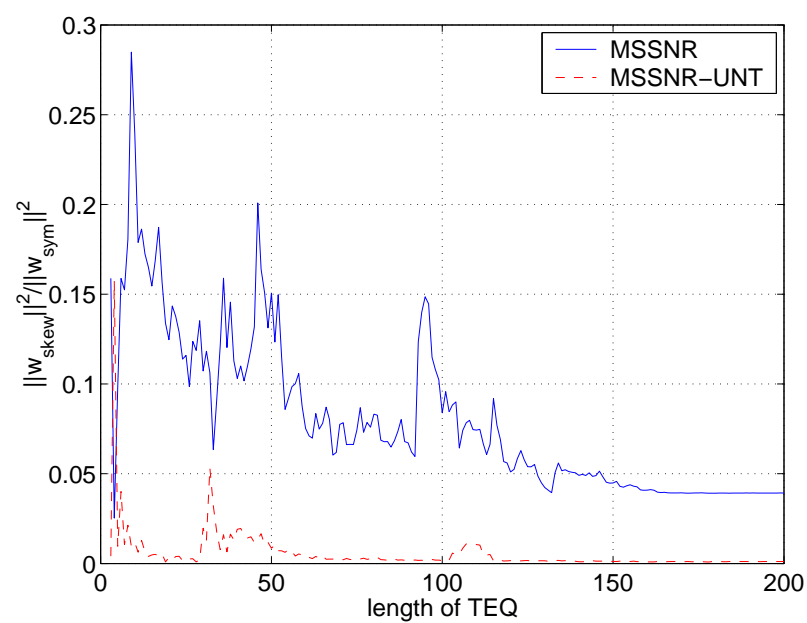

Fig. 2. Energy in the skew-symmetric part over the energy in the symmetric part of the TEQ. The data was delayoptimized and averaged over ADSL CSA test loops $1-8$.

are also eigenvectors with the same eigenvalue $\lambda$. Thus, for a given eigenpair $(\lambda, \mathbf{w})$, we can always force the eigenvector to be symmetric, $\mathbf{w}_{\text {sym }}=(\mathbf{w}+\mathbf{J w}) / 2$, or skewsymmetric, $\mathbf{w}_{\text {skew }}=(\mathbf{w}-\mathbf{J w}) / 2$, without changing the eigenvalue.

If all of the eigenvalues of $\mathbf{A}^{-1} \mathbf{B}$ are distinct, then its eigenvectors are unique. Thus, $\mathbf{w}, \mathbf{J} \mathbf{w}$, and $-\mathbf{J w}$ must all be identical (up to a scalar, such as -1 ). This requires each $\mathbf{w}$ to be either symmetric or skew-symmetric.

For the channel convolution matrix $\mathbf{H}$, we have $\mathbf{H}^{T} \mathbf{H} \in$ $V_{L_{w}}$. This suggests that $\mathbf{A}$ and $\mathbf{B}$ may also be in $V_{L_{w}}$, since $\mathbf{A}=\mathbf{H}_{\text {wall }}^{T} \mathbf{H}_{\text {wall }}$ and $\mathbf{B}=\mathbf{H}_{\text {win }}^{T} \mathbf{H}_{\text {win }}$, rather than $\mathbf{H}^{T} \mathbf{H}$. Unfortunately, $\mathbf{A}$ and $\mathbf{B}$ are not perfectly symmetric centrosymmetric, but they are approximately so. In addition, $\mathbf{A}$ is always invertible when the channel is longer than $\nu+1$ [13]. Thus, the eigenvectors of $\mathbf{A}$ and of $\mathbf{A}^{-1} \mathbf{B}$ will all be approximately symmetric or skew-symmetric. Furthermore, we can replace $\mathbf{A}$ by $\left(\mathbf{A}+\mathbf{R}_{n}\right)$ in Theorem 3.1 to obtain similar results for the MMSE case. Oddly enough, the finite length MSSNR and MSSNR-UNT TEQs always seem to be nearly symmetric rather than nearly skew-symmetric, and the point of symmetry is not in the center of the TEQ.

To quantify the symmetry of the MSSNR and MSSNRUNT TEQ designs for various parameter values, we computed both TEQs for $3 \leq L_{w} \leq 200$. For each TEQ, we decomposed $\mathbf{w}$ into $\mathbf{w}_{\text {sym }}$ and $\mathbf{w}_{\text {skew }}$, and then computed $\left\|\mathbf{w}_{\text {skew }}\right\|^{2} /\left\|\mathbf{w}_{\text {sym }}\right\|^{2}$. A plot of this ratio is shown in Fig. 2. The transmission delay in samples, $\Delta$, was determined via a global search for the MSSNR solution, and the same $\Delta$ was used for each corresponding MSSNR-UNT solution. The ratios were computed for Carrier Serving Area (CSA) test loops 1 through 8 and then averaged. Matlab code to reproduce Fig. 2 is available at [15].
The MSSNR-UNT TEQ becomes increasingly symmetric for large TEQ lengths, whereas the MSSNR TEQ is approximately symmetric for all lengths, but does not display as strong a trend. Symmetric TEQs can be initialized by only computing half of the TEQ coefficients. For MSSNR, MSSNR-UNT, and MMSE solutions, this reduces the problem from finding an eigenvector (or generalized eigenvector) of an $L_{w} \times L_{w}$ matrix to finding an eigenvector (or generalized eigenvector) of a $\left\lceil L_{w} / 2\right\rceil \times\left\lceil L_{w} / 2\right\rceil$ matrix [14]. This leads to a significant reduction in complexity, at the expense of throwing away the skew-symmetric portion of the filter. Reduced complexity algorithms are discussed in Section 4.

Yet another advantage of a perfectly symmetric TEQ is that is has a linear phase with known slope. Thus, if the channel is known, the phase response of the effective channel is known before the TEQ is designed. This allows the FEQ to be partially trained in parallel with the TEQ.

\subsection{Infinite length TEQ designs: asymptotic results}

This section examines the limiting behavior of $\mathbf{A}$ and $\mathbf{B}$, and the resulting limiting behavior of the eigenvectors of $\mathbf{A}$ (i.e. the MSSNR-UNT solution).

Theorem 3.2 For a channel convolution matrix $\mathbf{H}$ and $\mathbf{A}$ as in (2),

$$
\lim _{L_{w} \rightarrow \infty} \frac{\left\|\mathbf{H}^{T} \mathbf{H}-\mathbf{A}\right\|_{F}}{\|\mathbf{A}\|_{F}}=0
$$

where $\|\cdot\|_{F}$ denotes the Frobenius norm.

Sketch of proof: Under the assumptions

$$
\begin{aligned}
& \text { A1: } \Delta>L_{h}>\nu, \\
& \text { A2: } L_{w}>\Delta+\nu,
\end{aligned}
$$

we can partition $\mathbf{H}$ as

$$
\mathbf{H}=\left[\begin{array}{ccccc}
\mathbf{H}_{1} & \mathbf{H}_{L 2} & \mathbf{H}_{L 1} & \mathbf{0} & \mathbf{0} \\
\mathbf{0} & \mathbf{H}_{U 3} & \mathbf{H}_{M} & \mathbf{H}_{L 3} & \mathbf{0} \\
\mathbf{0} & \mathbf{0} & \mathbf{H}_{U 1} & \mathbf{H}_{U 2} & \mathbf{H}_{2}
\end{array}\right]
$$

The row blocks have heights $\Delta,(\nu+1)$, and $\left(L_{h}+L_{w}-\nu-\right.$ $\Delta)$; and the column blocks have widths $\left(\Delta-L_{h}\right),(\nu+1)$, $\left(L_{h}-\nu-1\right),(\nu+1)$, and $\left(L_{w}-\nu-\Delta\right)$. The sections $\left[\mathbf{H}_{L 2}, \mathbf{H}_{L 1}\right]$ and $\mathbf{H}_{L 3}$ are both lower triangular and contain the "head" of the channel, $\left[\mathbf{H}_{U 1}, \mathbf{H}_{U 2}\right]$ and $\mathbf{H}_{U 3}$ are both upper triangular and contain the channel "tail," $\mathbf{H}_{1}$ and $\mathbf{H}_{2}$ are tall channel convolution matrices, and $\mathbf{H}_{M}$ is Toeplitz. Then $\mathbf{H}_{\text {win }}$ is simply the middle row (of blocks) of $\mathbf{H}$, and $\mathbf{H}_{\text {wall }}$ is the concatenation of the top and bottom rows.

The limiting behavior for $\mathbf{B}=\mathbf{H}_{w i n}^{T} \mathbf{H}_{w i n}$ is

$$
\begin{aligned}
\mathbf{B} & =\left[\mathbf{0}, \mathbf{H}_{U 3}, \mathbf{H}_{M}, \mathbf{H}_{L 3}, \mathbf{0}\right]^{T}\left[\mathbf{0}, \mathbf{H}_{U 3}, \mathbf{H}_{M}, \mathbf{H}_{L 3}, \mathbf{0}\right] \\
& \triangleq\left[\mathbf{0}, \overline{\mathbf{H}}_{3}^{T}, \mathbf{0}\right]^{T}\left[\mathbf{0}, \overline{\mathbf{H}}_{3}^{T}, \mathbf{0}\right] .
\end{aligned}
$$


Table 1. Achievable bit rate (Mbps) for MSSNR and symmetric MSSNR designs, using 32-tap TEQs. The channel has AWGN but no crosstalk. The signal power was $23 \mathrm{dBm}$ and the noise power was $-140 \mathrm{dBm} / \mathrm{Hz}$. The CSA channels can be obtained at [16].

\begin{tabular}{|c|c|c|c|}
\hline Loop \# & MSSNR & SYM-MSSNR & loss \\
\hline CSA1 & 12.187 & 10.921 & $10.39 \%$ \\
CSA2 & 13.016 & 12.493 & $4.02 \%$ \\
CSA3 & 11.543 & 11.529 & $0.12 \%$ \\
CSA4 & 11.696 & 11.431 & $2.27 \%$ \\
CSA5 & 12.120 & 11.800 & $2.64 \%$ \\
CSA6 & 10.995 & 10.798 & $1.79 \%$ \\
CSA7 & 10.978 & 10.880 & $0.89 \%$ \\
CSA8 & 10.294 & 9.956 & $3.28 \%$ \\
\hline
\end{tabular}

As $L_{w}$ and $\Delta$ increase, the only change to $\mathbf{B}$ is the size of the zero matrices. It can be shown that

$$
\|\mathbf{B}\|_{F}^{2}=\left\|\overline{\mathbf{H}}_{3} \overline{\mathbf{H}}_{3}^{T}\right\|_{F}^{2} \leq\|\mathbf{h}\|_{2}^{4} \cdot\left(\nu+L_{h}\right)^{2},
$$

where $L_{h}$ is the channel length.

Since $\mathbf{A}=\mathbf{H}_{\text {wall }}^{T} \mathbf{H}_{\text {wall }}$, it becomes a $5 \times 5$ block matrix, with $\mathbf{A}_{1,1}=\mathbf{H}_{1}^{T} \mathbf{H}_{1}$ and $\mathbf{A}_{5,5}=\mathbf{H}_{2}^{T} \mathbf{H}_{2}$. Thus,

$$
\begin{aligned}
\|\mathbf{A}\|_{F}^{2} & \geq\left\|\mathbf{H}_{1}^{T} \mathbf{H}_{1}\right\|_{F}^{2}+\left\|\mathbf{H}_{2}^{T} \mathbf{H}_{2}\right\|_{F}^{2} \\
& \geq\|\mathbf{h}\|_{2}^{4} \cdot\left(L_{w}-L_{h}-\nu\right) .
\end{aligned}
$$

Noting that $\mathbf{B}=\mathbf{H}^{T} \mathbf{H}-\mathbf{A}$, taking the ratio of (12) to (13) and taking the limit completes the proof.

Theorem 3.2 suggests (heuristically) that in the limit, the eigenvectors of $\mathbf{A}$ (the MSSNR-UNT solution) converge to the eigenvectors of $\mathbf{H}^{T} \mathbf{H}$. Since $\mathbf{H}^{T} \mathbf{H} \in V_{L_{w}}$, its eigenvectors are symmetric or skew-symmetric.

\section{SYMMETRIC TEQ ALGORITHM}

We can force a perfectly symmetric even-length TEQ by rewriting $\mathbf{w}^{T} \mathbf{A} \mathbf{w}$ as

$$
\begin{aligned}
& {\left[\mathbf{v}^{T}, \mathbf{v}^{T} \mathbf{J}\right]\left[\begin{array}{ll}
\mathbf{A}_{11} & \mathbf{A}_{12} \\
\mathbf{A}_{21} & \mathbf{A}_{22}
\end{array}\right]\left[\begin{array}{c}
\mathbf{v} \\
\mathbf{J} \mathbf{v}
\end{array}\right]} \\
& =\mathbf{v}^{T} \underbrace{\left[\mathbf{A}_{11}+\mathbf{J} \mathbf{A}_{21}+\mathbf{A}_{12} \mathbf{J}+\mathbf{J} \mathbf{A}_{22} \mathbf{J}\right]}_{\hat{\mathbf{A}}} \mathbf{v}
\end{aligned}
$$

with an analogous definition of $\hat{\mathbf{B}}$. The MSSNR problem is reduced to

$$
\min _{\mathbf{v}}\left(\mathbf{v}^{T} \hat{\mathbf{A}} \mathbf{v}\right) \text { subject to } \mathbf{v}^{T} \hat{\mathbf{B}} \mathbf{v}=1
$$

Note that $\mathbf{A}$ and $\mathbf{B}$ have dimensions $L_{w} \times L_{w}$, whereas $\hat{\mathbf{A}}$ and $\hat{\mathbf{B}}$ have dimensions $\frac{L_{w}}{2} \times \frac{L_{w}}{2}$. We still require a symmetric generalized eigendecomposition, but its complexity has been reduced by a factor of 4 .
Table 1 shows the achievable bit rate using a 32-tap TEQ, for the MSSNR method [4] and the proposed symmetric MSSNR method. The channels were the eight standard CSA test loops. The performance loss for the proposed algorithm ranges from $0.1 \%$ (loop 3) to $10 \%$ (loop 1), with an average loss of $3 \%$. For some TEQ lengths (not shown), the symmetric TEQs have higher bit rates than their unconstrained counterparts. The symmetric MSSNR TEQ design has been implemented in the DMT TEQ Toolbox [16].

\section{REFERENCES}

[1] D. D. Falconer and F. R. Magee, "Adaptive Channel Memory Truncation for Maximum Likelihood Sequence Estimation," Bell Sys. Tech. Journal, pp. 1541-1562, Nov. 1973.

[2] J. S. Chow, J. M. Cioffi, and J. A. C. Bingham, "Equalizer Training Algorithms for Multicarrier Modulation Systems," in Proc. IEEE Int. Conf. on Comm., May 1993, pp. 761-765.

[3] N. Al-Dhahir and J. M. Cioffi, "Optimum Finite-Length Equalization for Multicarrier Transceivers," IEEE Trans. on Comm., vol. 44, no. 1, pp. 56-64, Jan. 1996.

[4] P. J. W. Melsa, R. C. Younce, and C. E. Rohrs, "Impulse Response Shortening for Discrete Multitone Transceivers," IEEE Trans. on Comm., vol. 44, pp. 1662-1672, Dec. 1996.

[5] M. Nafie and A. Gatherer, "Time-Domain Equalizer Training for ADSL," in Proc. IEEE Int. Conf. on Comm., Montreal, Canada, June 1997, vol. 2, pp. 1085-1089.

[6] R. Schur and J. Speidel, "An Efficient Equalization Method to Minimize Delay Spread in OFDM/DMT Systems," in IEEE International Conference on Communications, June 2001.

[7] N. Lashkarian and S. Kiaei, "Optimum Equalization of Multicarrier Systems: A Unified Geometric Approach," IEEE Trans. on Comm., vol. 49, pp. 1762-1769, Oct. 2001.

[8] G. Arslan, B. L. Evans, and S. Kiaei, "Equalization for Discrete Multitone Receivers To Maximize Bit Rate," IEEE Trans. on Signal Processing, vol. 49, no. 12, pp. 3123-3135, Dec. 2001.

[9] R. K. Martin, J. Balakrishnan, W. A. Sethares, and C. R. Johnson, Jr., "A Blind, Adaptive TEQ for Multicarrier Systems," IEEE Signal Processing Letters, Nov. 2002.

[10] M. Moonen, G. Cuypers, K. Vanbleu, and G. Ysebaert, "ADSL Pertone Equalizer Design Issues - A Status Report," in Proc. ThirtySixth Asilomar Conf. on Signals, Systems, and Computers, 2002.

[11] C. Yin and G. Yue, "Optimal Impulse Response Shortening for Discrete Multitone Transceivers," Electronics Letters, vol. 34, pp. 3536, Jan. 1998.

[12] D. Daly, C. Heneghan, and A. D. Fagan, "A Minimum Mean-Squared Error Interpretation of Residual ISI Channel Shortening for Discrete Multitone Transceivers," in Proc. IEEE Int. Conf. on Acoustics, Speech, and Signal Processing, May 2001, vol. 4, pp. 2065-2068.

[13] R. K. Martin, M. Ding, B. L. Evans, and C. R. Johnson, Jr., "Infinite Length Results and Design Implications for Time-Domain Equalizers," Submitted to IEEE Trans. on Signal Processing, Oct. 2002.

[14] A. Cantoni and P. Butler, "Eigenvalues and Eigenvectors of Symmetric Centrosymmetric Matrices," Linear Algebra and Its Applications, vol. 13, pp. 275-288, 1976.

[15] R. K. Martin, "Matlab code for papers by R. K. Martin," available at http://bard.ece.cornell.edu/matlab/martin/index.html .

[16] G. Arslan, M. Ding, B. Lu, Z. Shen, and B. L. Evans, "TEQ design toolbox," The University of Texas at Austin, http://www.ece.utexas.edu/ bevans/projects/adsl/dmtteq/dmtteq.html. 